\title{
Interatomic Coulombic electron capture in atomic, molecular, and quantum dot systems
}

\author{
Annika Bande ${ }^{a}$, Federico M. Pont, Kirill Gokhberg and Lorenz S. Cederbaum \\ Theoretische Chemie, Physikalisch-Chemisches Institut, Universität Heidelberg, Im Neuenheimer Feld \\ 229, 69120 Heidelberg, Germany
}

\begin{abstract}
The interatomic Coulombic electron capture (ICEC) process has recently been predicted theoretically for clusters of atoms and molecules. For an atom $A$ capturing an electron $e(\varepsilon)$ it competes with the well known photorecombination, because in an environment of neutral or anionic neighboring atoms $B, A$ can transfer its excess energy in the ultrafast ICEC process to $B$ which is then ionized. The cross section for $e(\varepsilon)+$ $A+B \rightarrow A^{-}+B^{+}+e\left(\varepsilon^{\prime}\right)$ has been obtained in an asymptotic approximation based on scattering theory for several clusters [1, 2]. It was found that ICEC starts dominating the PR for distances among participating species of nanometers and lower. Therefore, we believe that the ICEC process might be of importance in the atmosphere, in biological systems, plasmas, or in nanostructured materials. As an example for the latter, ICEC has been investigated by means of electron dynamics in a model potential for semiconductor double quantum dots (QDs) [3]. In the simplest case one QD captures an electron while the outgoing electron is emitted from the other. The reaction probability for this process was found to be relatively large.
\end{abstract}

\section{Introduction}

Dissociative recombination (DR) is a process in which a molecular ion captures an electron and then dissociates as result of the charge uptake. The process has been observed over years in environments as atmospheric, interstellar space, combustion, or laboratory plasmas [4-6] in which free electrons are present. Other electron capture processes known are photon based, namely the photorecombination (PR, i.e. radiative attachment/recombination $[7,8]$ ) in which a photon is emitted as result of the capture as well the dielectronic recombination, which is a special case of PR proceeding through an electronic resonance [9].

In some of these environments molecules that can undergo DR coexist with other atomic or molecular species in the distance of nanometers. For such environments we have recently proposed a new mechanism, the interatomic Coulombic electron capture (ICEC), that might be competing with DR or at least PR and should not be overlooked [1, 2]. During ICEC an electron is taken up by one neutral or positively charged atom or molecule $A$ (Fig. 1, left). The binding and excess energy of the captured electron is transferred, via long-range Coulomb interaction, to a neighboring atom or molecule

\footnotetext{
${ }^{a}$ Corresponding author: Annika.Bande@pci.uni-heidelberg.de
}

This is an Open Access article distributed under the terms of the Creative Commons Attribution License 4.0, which permits unrestricted use, distribution, and reproduction in any medium, provided the original work is properly cited. 


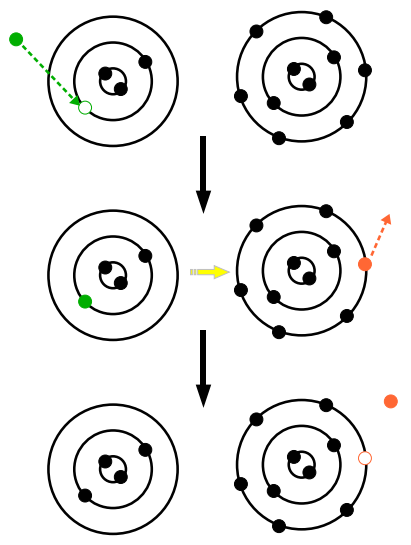

Figure 1. Schematic representation of ICEC in a cluster of two different atoms $A$ (left) and $B$ (right). $A$ captures an electron (green) with a given energy $\varepsilon$. At the same time energy is transferred to $B$ (yellow arrow) from which an electron with a different energy $\varepsilon^{\prime}$ (orange) is emitted.

$B$ (right), which is then ionized according to

$$
e(\varepsilon)+A+B \rightarrow A^{-}+B^{+}+e\left(\varepsilon^{\prime}\right) .
$$

Depending on the state of $A$ into which the electron is captured or the number of participating electrons we distinguish among ICEC with capture into the ground (Sect. 2.1) or excited (Sect. 2.2) state and the dielectronic ICEC (dICEC, Sect. 2.3) that we have studied by multichannel scattering theory [1, 2, 10].

Another theory that we employed was exact electron dynamics which we utilized to prove ICEC to be a possible process in nanoscale semiconductor quantum dots (QDs, Sect. 3, [3]).

\section{ICEC in atoms and molecules}

\subsection{ICEC through ground states}

An isolated neutral or ionic atom $A$ can take up one electron solely by emission of a photon, which is known as PR [7, 8]. However, when the atom is embedded in an environment of one or several neighboring neutral or ionic atoms or molecules $B$ ICEC can occur (Eq. (1)). The energy conservation

$$
E A^{(A)}+\varepsilon=I P^{(B)}+\varepsilon^{\prime}=E_{v p h}
$$

among the electron affinity $E A^{(A)}$ of $A$ and the ionization potential $I P^{(B)}$ of $B$ defines the energy of the virtual photon transferred from $A$ to $B[1,2]$. The virtual photon mechanism allows to rationalize the process in terms of cross sections for intermediate steps. Although the energy threshold for the incoming electron in PR is simply $\varepsilon^{t}=0$, it is conditional for ICEC through

$$
\varepsilon^{t}=\left\{\begin{array}{l}
0, \text { if } I P^{(B)}<E A^{(A)} \\
\text { else: } I P^{(B)}-E A^{(A)} .
\end{array}\right.
$$

The ICEC cross section

$$
\sigma_{\mathrm{ICEC}}(\varepsilon)=N \frac{3 \hbar^{4} c^{4}}{4 \pi} \frac{\sigma_{\mathrm{PI}}^{(\mathrm{B})}\left(\varepsilon^{\prime}\right)}{R^{6} E_{v p h}^{4}} \sigma_{\mathrm{PR}}^{(\mathrm{A})}(\varepsilon)
$$


was derived in terms of multichannel scattering theory. Equation (4) says that ICEC dominates PR for short interatomic distances $R$, for small virtual photon energies $E_{v p h}$, i.e. slow incoming electrons or higher excited states (inverse to PR), for large photoionization (PI) cross sections $\sigma_{\mathrm{PI}}^{(\mathrm{B})}$ of $B$ (e.g. $B$ is molecule with shape resonance), and for a higher number $N$ of neighbors [1,2].

An example for the efficiency of ICEC is the halogen-halide reaction

$$
e(\varepsilon)+B r+\mathrm{Cl}^{-} \rightarrow \mathrm{Br}^{-}+\mathrm{Cl}+e\left(\varepsilon^{\prime}\right)
$$

with threshold energy $\varepsilon^{t}=0.228 \mathrm{eV}$ (or $\varepsilon^{t}=0 \mathrm{eV}$ for the respective backwards reaction) when using $E A^{(B r)}=3.313 \mathrm{eV}$ and $I P^{\left(C l^{-}\right)}=3.601 \mathrm{eV}[11]$. A reaction with several neighbors $(N=1,6)$ that we studied was [1]:

$$
e(\varepsilon)+\mathrm{Mg}^{2+}+\mathrm{N} \mathrm{H}_{2} \mathrm{O} \rightarrow \mathrm{Mg}^{+}+\mathrm{H}_{2} \mathrm{O}^{-}+(\mathrm{N}-1) \mathrm{H}_{2} \mathrm{O}+e\left(\varepsilon^{\prime}\right) .
$$

\subsection{ICEC through excited states}

Contrary to PR ICEC becomes more likely with small virtual photon energies $E_{v p h}$ and thus when capture is into excited virtual orbitals of $A[1,2,7,8]$. Examples for this are the capture of an electron by different noble gas cations into their $N e\left(2 p^{-1} 3 p\right)$ or the $H e\left(1 s^{-1} 2 p\right)$ state that undergo ICEC with other noble gas atoms or benzene as neighbor [2]. The ICEC cross section was observed to be larger than the PR cross section for all incoming electron energies in the case of the excited state, whereas it was smaller for large $\varepsilon$ in the case of the ground state. It is noteworthy that an energy threshold exists for ICEC through excited states due to Eq. (3) which is for $N e\left(2 p^{-1} 3 p\right)$ with $E A^{\left(N e^{+}\right)}=2.943 \mathrm{eV}$ $\varepsilon^{t}=6.51 \mathrm{eV}$ and $\varepsilon^{t}=10.50 \mathrm{eV}$, respectively, when the neighbors are Benzene $\left(I P^{(B z)}=9.45 \mathrm{eV}[12]\right)$ or Xenon $\left(I P^{(X e)}=12.13 \mathrm{eV}[13]\right)$. Another example that has been studied in detail for the merit of very small virtual photon energies is [2]

$$
e(\varepsilon)+M g^{+}+B r^{-} \rightarrow M g+B r+e\left(\varepsilon^{\prime}\right) .
$$

\subsection{Dielectronic ICEC}

Electron capture into virtual states does not only allow for single-electron, but also for dielectronic processes. In case of isolated cations, dielectronic recombination has been proved in systems where the electron is captured into a Rydberg state by exciting one of the bound electrons which then relaxes via photon emission [9]. The corresponding dICEC process is combined of the aforementioned dielectronic capture process and the interatomic Coulombic decay (ICD [14-17]) process [2]. In ICD the excited electron on the moiety $A$ relaxes by transferring a virtual photon to a neighbor $B$ which then emits an electron. The balance equation for the total dICEC process reads

$$
e(\varepsilon)+A+B \rightarrow\left(A^{-}\right)^{* *}+B \rightarrow\left(A^{-}\right)^{*}+B^{+}+e\left(\varepsilon^{\prime}\right)
$$

and the dICEC cross section

$$
\sigma_{d I C E C}=\frac{\pi}{k_{p h}^{2}} \frac{g_{d}}{g_{i n}} \frac{\Gamma_{p h} \Gamma_{I C D}}{\left(\varepsilon-\varepsilon_{R}\right)^{2}+\Gamma^{2} / 4}
$$

was derived along the same lines as before [2]. Here, $g_{d}$ and $g_{i n}$ are the weights of the decaying and the initial state, respectively, $k_{p h}$ is the absolute of the wave vector of the emitted photon, and $\varepsilon_{R}$ is the resonance energy of the decaying state. The total widths $\Gamma=\Gamma_{A I}+\Gamma_{p h}+\Gamma_{I C D}$ is composed of the 

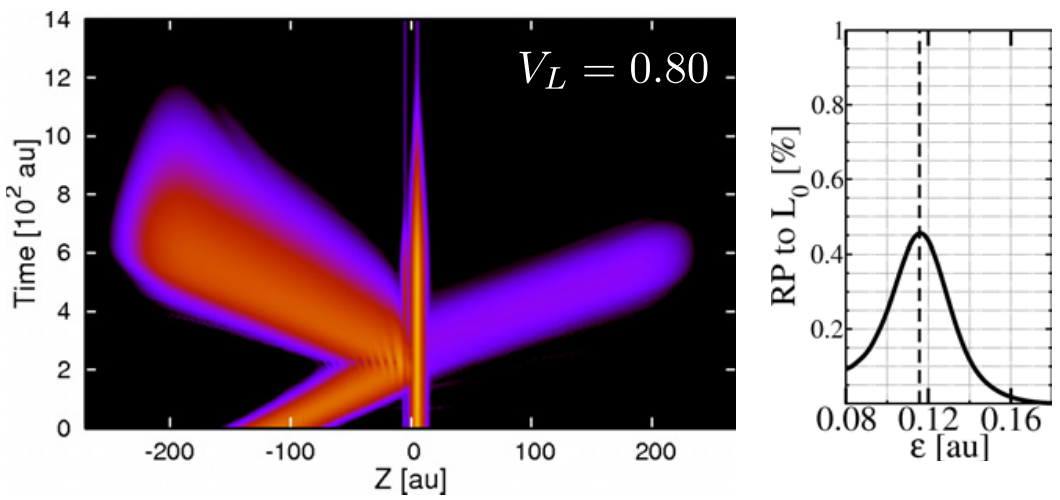

Figure 2. Evolution of the electronic density (left panel) and the corresponding RP (right panel) for the model potential defined by $R=10$ a.u., $V_{L}=0.80$ a.u., $V_{R}=0.6$ a.u., $b_{L, R}=1.0$ a.u., $E_{L_{0}}=-0.3769$ a.u., $E_{R_{0}}=$ -0.2463 a.u. and the initial WP defined by $x=-100$ a.u., $\varepsilon_{i}=0.130$ a.u., $\Delta x_{W P_{i}}=10$ a.u., and $\Delta E_{W P_{i}}=0.051$ a.u. The incoming wave packet approaches the DQD from the left. The single one-electron state $L_{0}$ is occupied by the incoming electron and emission of the electron initially located in $R_{0}$ through ICEC takes place starting at about 200 a.u. in time. The energy conservation dictates that the emitted packet acquires momentum and is clearly seen from its flatter slope of the packet $\left(\varepsilon_{f}=0.2606\right.$ a.u.). The RP is peaked at $\varepsilon_{i}^{(\text {peak })}=-E_{R_{0}}-\Delta E=0.1157$ a.u. indicated by a dashed vertical line.

autoionization, photon, and the ICD widths of which the latter is given as

$$
\Gamma_{I C D}=N \frac{3 c^{4} \hbar^{4}}{4 \pi} \frac{\sigma_{\mathrm{PI}}^{(\mathrm{B})}(\varepsilon) \Gamma_{p h}}{E_{v p h}^{4} R^{6}} .
$$

It is obtained using the multipole expansion of the electron-electron interaction term which couples the electronic motion on the centers $A$ and $B$ and drives the interatomic decay [18, 19]. Equations (9) and (10) mean that dICEC also dominates for small $R$ and $E_{v p h}$. However, since dICEC is a dielectronic process involving a resonance decay, capture only into discrete high-energy states has been shown for the examples $N e^{+}\left(2 p^{-1}\right) \rightarrow N e^{* *}\left(2 s^{-1} n p\right) \rightarrow N e\left(2 p^{-1} n p\right), H e^{+}\left(2 p^{-1}\right) \rightarrow H e^{* *}(2 s n p+2 p n s) \rightarrow$ $H e\left(2 p^{-1} n p\right)$, and $M^{+} \rightarrow M g^{* *}(3 p n s+3 p n d)$ [2]. In all cases $\sigma_{d I C E C}$ as a function of $\varepsilon$ gives a line spectrum with usually increasing line intensity for larger $\varepsilon$ when $\Gamma_{I C D}$ dominates $\Gamma_{p h}$. Moreover, if the autoionization rate is large then it dominates $\Gamma_{I C D}$ and lines are less intense [2].

\section{ICEC in quantum dots}

There are two main components involved in ICEC, namely the two binding entities $A$ and $B$ (previously atoms or molecules, Sect. 2) and two mobile entities (electrons). The process is affected by the binding energy and symmetry of the electronic states in the atoms, but it does not require the specific atomelectron interaction to achieve the capture. The most relevant contribution to ICEC comes from the electron correlation between electrons located in different moieties, leaving some space for the binding potential to be generalized. Thus electrons can be localized in binding potentials that are commonly used to theoretically describe nanometer sized semiconductor structures called quantum dots (QDs) also known as artificial atoms. The name was coined by Kastner et al. [20] after the discovery of quantized electronic levels inside the QDs. The amount of experiments performed in QDs and the variety of phenomena with a straight correlation to those found in atoms increased enormously over the years [21-29] and was mainly motivated by the vast range of technological applications of QDs as 
third generation solar cells [30, 31], light emitting diodes [32, 33], quantum information processing [25], or infrared photo detectors [34-36] among others.

The ICEC process first described in atoms was found to be also present in QDs [3]. The natural configuration to prove ICEC in QDs is a pair of neighboring QDs usually referred to as a double quantum dot (DQD). The dots are chosen to be separate enough to keep individual characteristics of the dots and close enough to allow the confined electrons inside each of the dots to interact with electrons in the neighboring dot via long-range Coulomb interaction. We have performed studies of ICEC in QDs using a model potential for a nanowire with a DQD defined along it [3]. Analogue models have been used by some of us to prove ICD [37-40] as another long-range correlation driven mechanism inside DQDs.

We used electron dynamics to prove ICEC in DQDs [3]. The approach goes beyond the scattering tools used to describe ICEC in atoms (Sect. 2) and allows for a deeper understanding of the process as such. New physical insight into the intermediate steps of ICEC can be obtained and thus new phenomena be discovered. In our calculations we solved the time-dependent Schrödinger equation in a complete state space description by using the multiconfiguration time-dependent Hartree (MCTDH) method [41, 42] as implemented in the MCTDH suite of programs [43, 44].

Details on our investigations on ICEC in DQDs are published elsewhere [3]. One example is, however, presented in Fig. 2. We found that ICEC processes are particularly fast and can play a significant role in the dynamics contributing to the energy transfer between QDs. The ICEC mechanisms in DQDs are feasible to be implemented and exploited in devices using low energy electrons and confined directionality.

\section{Conclusion}

Our approaches to investigate the interatomic Coulombic electron capture process have been two-fold: we have studied atomic systems in an asymptotic approach based on multichannel-scattering theory and obtained general results on the ICEC cross section that were then used to study several systems. Here we found that ICEC can dominate PR for low $R$, low $E_{v p h}$, and high $N$ and that the dielectronic dICEC process is an alternative capture process at discrete, higher energies but otherwise similar conditions than ICEC itself.

The basic physics of the general long-range correlation induced energy transfer process has been understood by using fully-correlated electron dynamics in general binding potentials. From this approach we have learned about the importance of ICEC in semiconductor quantum dots in which it happens to a relatively high probability and can overcome other important capture mechanisms. Therefore we comprehend our results as an important contribution to state-of-the-art nanotechnology for the development of QD devices that generate monochromatic low energy electrons in given direction.

All findings made clear that ICEC is an important process of high generality which has been overlooked until now although it applies in very different environments as the atmosphere, plasmas, biological systems, and nanomaterials. With this presentation we hope to stimulate exciting experiments on ICEC.

Finally we would like to acknowledge financial support by the University of Heidelberg and the German Academic Exchange Service (DAAD).

\section{References}

[1] K. Gokhberg, L.S. Cederbaum, J. Phys. B: At. Mol. Opt. Phys. 42, 4 (2009)

[2] K. Gokhberg, L.S. Cederbaum, Phys. Rev. A. 82, 052707 (2010)

[3] F.M. Pont, A. Bande, L.S. Cederbaum, Phys. Ref. B 88, 241304(R) (2013) 
[4] D.R. Bates, Phys. Rev. 78, 492 (1950)

[5] S.L. Guberman, J. Phys. Conf. Ser. 300, 012001 (2011)

[6] R. Johnsen, J. Phys. Conf. Ser. 300, 012002 (2011)

[7] A. Müller, Adv. At. Mol. Opt. Phys. 55, 293 (2008)

[8] H.A. Bethe, E.E. Salpeter, Quantum Mechanics of One- and Two-Electron Atoms (Dover, New York, 2008)

[9] H.S.W. Massey, D.R. Bates, Rep. Prog. Phys. 9, 42 (1942)

[10] J.P. Taylor, Scattering Theory (Dover, New York, 2006)

[11] A. Mandl, Phys. Rev. A 14, 345 (1976)

[12] R. Feng, G. Cooper, C.E. Brion, J. Electron Spectrosc. 123, 199 (2002)

[13] C. Duzy, H.A. Hyman, Phys. Rev. A 22, 1878 (1980)

[14] S. Barth, S. Joshi, S. Marburger, V. Ulrich, A. Lindblad, G. Öhrwall, O. Björneholm, U. Hergenhahn, J. Chem. Phys. 122, 241102 (2005)

[15] T. Aoto, K. Ito, Y. Hikosaka, E. Shigemasa, F. Penent, P. Lablanquie, Phys. Rev. Lett. 97, 243401 (2006)

[16] K. Gokhberg, A.B. Trofimov, T. Sommerfeld, L.S. Cederbaum, Europhys. Lett. 72, 228 (2005)

[17] K. Gokhberg, V. Averbukh, L.S. Cederbaum, J. Chem. Phys. 124, 144315 (2006)

[18] V. Averbukh, I.B. Müller, L.S. Cederbaum, Phys. Rev. Lett. 93, 263002 (2004)

[19] K. Gokhberg, S. Kopelke, N.V. Kryzhevoi, P. Kolorenč, L.S. Cederbaum, Phys. Rev. A 81, 013417 (2010)

[20] M.A. Kastner, Phys. Today 46, 24 (1993)

[21] W.G. van der Wiel, S. De Franceschi, J.M. Elzerman, T. Fujisawa, S. Tarucha, L.P. Kouwenhoven, Rev. Mod. Phys. 75, 1 (2002)

[22] J. Salfi, S. Roddaro, D. Ercolani, L. Sorba, I. Savelyev, M. Blumin, H.E. Ruda, F. Beltram, Semicond. Sci. Technol. 25, 024007 (2010)

[23] E.A. Laird, J.M. Taylor, D.P. DiVincenzo, C.M. Marcus, M.P. Hanson, A.C. Gossard, Phys. Rev. B 82, 075403 (2010)

[24] S. Roddaro, A. Pescaglini, D. Ercolani, L. Sorba, F. Beltram, Nano Lett. 11, 1695 (2011)

[25] S. Nadj-Perge, V.S. Pribiag, J.W.G. van den Berg, K. Zuo, S.R. Plissard, E.P.A.M. Bakkers, S.M. Frolov, L.P. Kouwenhoven, Phys. Rev. Lett. 108, 166801 (2012)

[26] T. Fujisawa, T.H. Oosterkamp, W.G. van der Wiel, B.W. Broer, R. Aguado, S. Tarucha, K.L. P., Science 282, 932 (1998)

[27] A. Shabaev, A.L. Efros, A.J. Nozik, Nano Lett. 6, 2856 (2006)

[28] K. Müller, A. Bechtold, C. Ruppert, M. Zecherle, G. Reithmaier, M. Bichler, H.J. Krenner, G. Abstreiter, A.W. Holleitner, J.M. Villas-Boas et al., Phys. Rev. Lett. 108, 197402 (2012)

[29] M. Benyoucef, V. Zuerbig, J.P. Reithmaier, T. Kroh, A.W. Schell, T. Aichele, O. Benson, Nanoscale Res. Lett. 7, 493 (2012)

[30] A.J. Nozik, M.C. Beard, J.M. Luther, M. Law, R.J. Ellingson, J.C. Johnson, Chem. Rev. 110, 6873 (2010)

[31] J. Jean, S. Chang, P.R. Brown, J.J. Cheng, P.H. Rekemeyer, M.G. Bawendi, S. Gradečak, V. Bulović, Adv. Mater. 25, 2790 (2013)

[32] B.S. Mashford, M. Stevenson, Z. Popovic, C. Hamilton, Z. Zhou, C. Breen, J. Steckel, V. Bulović, M.G. Bawendi, S. Coe-Sullivan et al., Nat Photon 7, 407 (2013)

[33] V. Wood, M.J. Panzer, J.M. Caruge, J.E. Halpert, M.G. Bawendi, V. Bulović, Nano Lett. 10, 24 (2010)

[34] S. Maimon, E. Finkman, G. Bahir, S.E. Schacham, J.M. Garcia, P.M. Petroff, Appl. Phys. Lett. 73, 2003 (1998)

[35] L. Chu, A. Zrenner, G. Böhm, G. Abstreiter, Appl. Phys. Lett. 75, 3599 (1999)

[36] S.F. Tang, S.Y. Lin, S.C. Lee, Appl. Phys. Lett. 78, 2428 (2001) 
[37] A. Bande, K. Gokhberg, L.S. Cederbaum, J. Chem. Phys. 135, 144112 (2011)

[38] A. Bande, K. Gokhberg, N. Moiseyev, L.S. Cederbaum, J. Phys.: Conf. Ser. 388, 152026 (2012)

[39] A. Bande, F.M. Pont, P. Dolbundalchok, K. Gokhberg, L.S. Cederbaum, EPJ Web Conf. 41, 04031 (2013)

[40] A. Bande, J. Chem. Phys. 138, 214104 (2013)

[41] H.D. Meyer, U. Manthe, L.S. Cederbaum, Chem. Phys. Lett. 165, 73 (1990)

[42] U. Manthe, H.D. Meyer, L.S. Cederbaum, J. Chem. Phys. 97, 3199 (1992)

[43] M.H. Beck, A. Jäckle, G.A. Worth, H.D. Meyer, Phys. Rep. 324, 1 (2000)

[44] H.D. Meyer, F. Gatti, G.A. Worth, eds., Multidimensional Quantum Dynamics (Whiley-VCH, 2009) 